\title{
The Pharmacology of Buprenorphine Microinduction for Opioid Use Disorder
}

\author{
Joao P. De Aquino $0^{1,2}$ (D) Suprit Parida ${ }^{1,2} \cdot$ Mehmet Sofuoglu ${ }^{1,2}$ \\ Accepted: 22 March 2021 / Published online: 5 April 2021 \\ (c) The Author(s), under exclusive licence to Springer Nature Switzerland AG 2021
}

\begin{abstract}
Although expanding the availability of buprenorphine—a first-line pharmacotherapy for opioid-use disorder (OUD)—has increased the capacity of healthcare systems to offer treatment, starting this medication is fraught with significant barriers. Standard induction regimens require persons with OUD to taper and discontinue full opioid agonists and experience opioid withdrawal prior to the first dose of buprenorphine. Further, emerging evidence indicates that precipitated withdrawal during induction may impact long-term treatment outcomes. Microinduction is a novel approach that, by harnessing buprenorphine's unique pharmacological profile, may allow circumventing the needed for prolonged opioid tapers, and reduce the risk of precipitated withdrawal-holding promise to enhance treatment access. In this review, we examine the pharmacological basis for microinduction and appraise the evidence of this approach to improve clinical outcomes among persons with OUD. First, we highlight the potential dose-dependent effects of buprenorphine on two key neuroadaptations at the mu-opioid receptor (MOR) - resensitization and upregulation. We then focus on how microinduction may reverse these chronic MOR neuroadaptations, allowing the maintenance of an adequate opioid tone, and thereby potentially circumventing opioid withdrawal. Second, we describe the clinical evidence available, derived from observational reports and open-label studies, examining the potential efficacy of microinduction. Despite significant heterogeneity - exemplified by variable buprenorphine formulations, daily doses, and schedules of administration - these data provide preliminary support for the feasibility of microinduction. Finally, we provide new mechanistic, methodological, and clinical insights to guide future translational research, as well as randomized, placebo-controlled clinical trials in this compelling agenda of pharmacotherapy development.
\end{abstract}

\section{Introduction}

As the toll of opioid-use disorder (OUD) has reached an epidemic scale, its full global impact is beginning to emerge. OUD causes mortality rates 15-20 times higher than those seen in the general population - the greatest burden of disease among all substance-use disorders [1,2]. According to the World Health Organization, approximately 27 million persons globally suffer from OUD and are in need of treatment [3]. The treatment for OUD consists of primarily of pharmacotherapy, which includes: methadone, a full a muopioid receptor (MOR) agonist; injectable extended-release

Joao P. De Aquino

joao.deaquino@yale.edu

1 VA Connecticut Healthcare System, 950 Campbell Avenue, 151D, West Haven, CT 06516, USA

2 Department of Psychiatry, Yale University School of Medicine, 300 George Street, New Haven, CT 06511, USA

\section{Key Points}

The induction onto buprenorphine for opioid-use disorder remains fraught with barriers, such as the need to experience opioid withdrawal.

Buprenorphine microinduction is a novel approach that may enhance treatment access, by harnessing buprenorphine's dose-dependent effects of mu-opioid receptor resensitization and upregulation, allowing the maintenance of an adequate opioid tone during the induction.

We integrate multiple lines of evidence to provide new mechanistic, methodological, and clinical insights, thereby guiding future studies investigating the therapeutic potential of buprenorphine microinduction. 
(IM XR) naltrexone, a MOR antagonist; and buprenorphine (BUP), a partial MOR agonist that is typically prescribed in combination with an orally inactive formulation of naloxone for OUD [4, 5]. As further described in Sect. 2.1, BUP has a unique pharmacological profile, characterized by its high affinity, partial agonism, and high potency at the MOR, as well as its ability to bind to the kappa-opioid receptors (KOR), where BUP acts as an antagonist. Despite the existence of three distinct pharmacotherapies for OUD, currently less than $10 \%$ of persons with OUD receive the much-needed treatment [6].

In recent years, an increase in the availability of BUP has extended the capacity of healthcare systems to treat persons with OUD [7]. Conveniently, unlike methadone-which can only be dispensed to treat OUD in Opioid Treatment Programs (OTPs), in countries like the USA-BUP can be provided by trained clinicians in office settings, and is typically subjected to less restrictive regulations. Further, in several European countries, BUP may be prescribed by general practitioners to treat OUD. The initiation-or induction-of pharmacotherapy with BUP, however, still presents a considerable challenge: due to its low intrinsic activity at the MOR, combined with its capacity to displace agonists from the MOR, BUP has the potential to acutely precipitate opioid withdrawal. Multiple studies have demonstrated that precipitated withdrawal during induction onto BUP occurs commonly, especially when it is preceded by recent exposure to full opioid agonists—-such as methadone or heroin-which is very often the case among persons with OUD. Emerging evidence indicates that in addition to the immediate physical and psychological discomfort, precipitated withdrawal during induction onto BUP may also impact long-term OUD treatment outcomes, such as treatment retention, as well as abstinence from non-medical opioid use [8,9].

To minimize the risk of precipitated withdrawal, therefore, patients who are exposed to full opioid agonists chronically are recommended to already be experiencing moderate withdrawal symptoms at the time of induction. A critical disadvantage of this approach is the requirement of patients who receive treatment with full opioid agonists to slowly taper and discontinue these medications-which may increase the risk of relapse and overdose. Further, by reducing the clinic visits that are needed to conduct the recommended full opioid agonist taper, major disruptions in the treatment of OUD caused by the SARS-CoV-2 pandemic have accentuated this challenge [10].

To circumvent this clinical dilemma and facilitate the transition from full opioid agonists-either prescribed or illicit-to BUP, an alternative approach is gaining popularity: microinduction. This approach entails administering very small doses of BUP, with gradual dose increases; meanwhile, the patient may continue to receive regular or fastertapering doses of a full opioid agonist, or may be exposed to illicit opioid agonists. Once a therapeutic dose of BUP is reached, the full opioid agonist is then promptly discontinued. This innovative method, thus, may have the compelling advantage of eliminating the need for a long taper or discontinuation of the full opioid agonist prior to the induction onto BUP-providing timely access to a first-line treatment for OUD. Further, as the risk of overdose is greater during the start and cessation of opioid pharmacotherapies, this approach may offer a safer way to transition from full opioid agonists to BUP, reducing the mortality associated with OUD [11]. Still, before microinduction can be implemented in clinical practice, the evidence supporting its feasibility and therapeutic potential warrants careful scrutiny.

The objective of this review is to critically appraise the pharmacological basis and the evidence of BUP microinduction strategies to enhance treatment outcomes among persons with OUD. First, we describe the pharmacology of BUP - focusing on its unique interaction with the MOR. Second, we analyze the challenges surrounding the traditional induction onto BUP, elaborating on the mechanistic and clinical rationale for microinduction. Third, we summarize the published studies and reports that used BUP microinduction methods. Lastly, we discuss gaps in knowledge and lay out directions for future research. Notably, this pharmacologically oriented review complements recent reviews on the clinical aspects of microinduction [12, 13]. We draw on our experience in Phase 1 human behavioral pharmacology studies and Phase 2 clinical trials to timely articulate the pharmacological principles underlying microinduction; rigorously appraise the available reports; and provide conceptual and methodological insights to advance this novel area of pharmacotherapy development.

\section{The Unique Pharmacology of Buprenorphine}

A semisynthetic opioid developed in the 1970s, BUP is a complex lipophilic molecule composed of multiple chiral centers, a morphine skeleton, and a unique cyclopropylmethyl group [14-16]. It binds to all four types of opioid receptors: mu-, delta- (DOR), KOR, and the opioid receptor-like 1 (ORL1) receptor. While MOR and ORL-1 mediate the rewarding and analgesic effects of opioids [17], DOR participate in mood-regulation [18] and KOR mediate analgesic, dysphoric, and psychotomimetic effects [14, 19].

BUP is an antagonist with a high binding affinity at the DOR and KOR, and an agonist with lower binding affinity at the ORL-1 [20]. The unique actions of BUP on multiple opioid receptors might explain its analgesic efficacy, antidepressant actions, and low sedative and psychotomimetic potential [21]. For the purposes of this review, we primarily focus on the pharmacological actions of BUP on the MOR, which are 
the primary mechanistic mediators of opioid dependence and withdrawal.

\subsection{Buprenorphine Interactions with Mu-Opioid Receptors}

Aside from BUP's actions on multiple opioid receptors, its unique pharmacological effects of result from: (1) high affinity to-and slow dissociation from-MOR; (2) partial agonism and low efficacy at MOR; and (3) high potency at MOR.

First, compared to full opioid agonists, BUP has a high binding affinity-or low Ki-for MOR [22]. For instance, the binding affinity of BUP for MOR is 5.4 and 6.2 times greater than that of morphine and fentanyl, respectively [23]. Further, BUP exhibits a slow dissociation from MOR in comparison with other opioids. This slow dissociation contributes to the prolonged effects of BUP, allowing oncea-day—or even less frequent-dosing, with minimal interdose withdrawal [20].

Second, as a partial agonist with low intrinsic efficacy at MOR, the maximal effects produced by BUP are lower than those produced by a full-or high efficacy-MOR agonist [24]. While in the presence of full opioid agonists, such as heroin or methadone, BUP acts like an opioid antagonist-competitively binding MOR, and thereby precipitating opioid withdrawal. Conversely, in the absence of MOR agonists, buprenorphine activates MOR partially-hence alleviating opioid withdrawal. Another clinical translation of the partial agonism of BUP is the ceiling effect for its MOR-mediated actions-such as euphoria, physiological dependence, and respiratory depression [25]. The relative infrequency of overdose deaths attributed to BUP, compared with those attributed to full opioid agonists, underscores its clinical safety [25].

Third, compared to other opioids, BUP has a high potency: The dose required to produce an effect of a given intensity-for instance, analgesia-is lower than that required from full opioid agonists. Specifically, converging preclinical and human studies have shown that BUP is 20-50 times more potent than morphine [26]. Importantly, the potency of a pharmacological agent depends on both its affinity and efficacy. When compared to morphine, relatively lower doses of BUP may elicit some degree of respiratory depression; however, higher doses of BUP, such as those used in clinical settings, do not lead to greater respiratory depression, given its lower MOR efficacy [26]. Further, as this example demonstrates, a simple conversion of BUP dose to morphine milligram equivalents (MME) may not adequately inform a transition from full opioid agonists to buprenorphine, or the assessment of overdose risk, solely based on the correspondent opioid dose.
In addition to the unique pharmacological profile of BUP, the structure and activity of the MOR itself may also influence their interactions. MOR are G-coupled protein receptors (GCPRs) - they are attached to inhibitory G-proteins (Goi), a family of proteins that act as molecular switches inside cells, transmitting signals from ligands outside the cell to its interior. G-proteins suppress neuronal activity by inhibiting adenylyl cyclase, reducing intracellular cyclic adenosine monophosphate (cAMP) levels, and regulating ion channels $[27,28]$. The ensuing cascade of signaling events limits neurotransmitter release and hyperpolarizes the cell membrane. Consistent with this, the rewarding and addictive effects of opioids are thought to be mediated by the hyperpolarization of GABAergic internerurons in the ventral tegmental area (VTA), which ultimately releases dopamine in the nucleus accumbens [27, 28]. Furthermore, opioids may cause the phosphorylation of specific amino acid residues on the cytoplasmic domain of MOR, recruiting a signaling cascade known as beta-arrestin pathway. Beta-arrestin is an adaptor protein that regulates receptor function and signal transduction activity. Recruitment of the beta-arrestin pathway causes MOR internalization through endocytosis, downregulation of MOR signaling [29-32], and, as a consequence, higher risk of opioid misuse, physiological dependence, and respiratory depression [27]. For a given opioid drug, the balance between G-protein and betaarrestin signaling (i.e., biased agonism) may determine the tradeoff between therapeutic efficacy and adverse effects [27]. BUP stimulates sufficient G-protein signaling, with limited recruitment of the beta-arrestin pathway-resulting in desirable bias toward MOR signaling and a better safety profile [27, 32].

In summary, the unique pharmacology of BUP generates an important therapeutic tradeoff: favorably, in the absence of full opioid agonists, BUP activates MOR enough to produce typical opioid effects-including analgesia and alleviation of opioid withdrawal — with a relatively wide therapeutic window. Unfavorably, when in the presence of full opioid agonists, however, BUP may displace them from MOR acutely, leading to an abrupt reduction of the opioid tone, manifested as precipitated withdrawal [33-36] (Fig. 1).

\section{Challenges Surrounding Induction onto Buprenorphine}

The induction onto BUP treatment still poses many clinical challenges. First, growing observational data has shown that induction onto BUP may precipitate withdrawal, potentially interfering with treatment retention and abstinence from non-medical opioid use $[8,9]$. Hence, ahead of the induction, patients are expected to already be experiencing moderate withdrawal symptoms. Yet, if patients are using a 


\section{Regular Interaction Between Buprenorphine and Full Opioid Agonist in Opioid-dependent Persons}

No Treatment

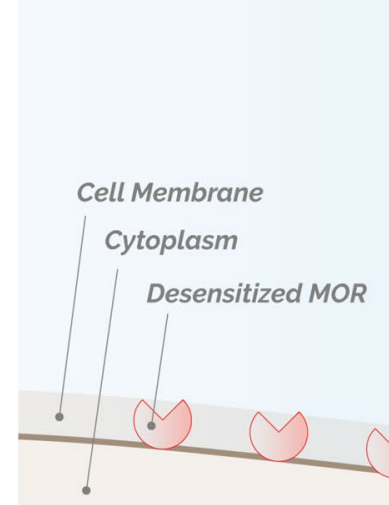

Downregulated MOR
Full MOR Agonist

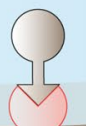

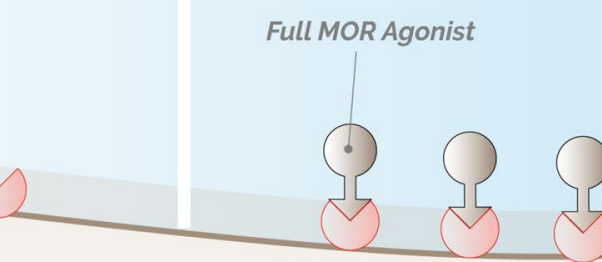

Full MOR Agonist + BUP

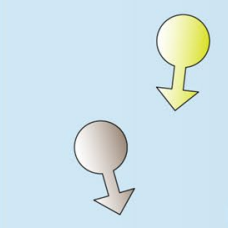

Buprenorphine

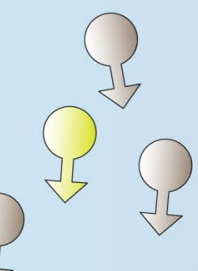

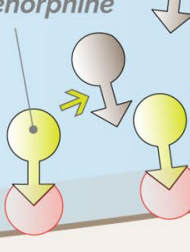

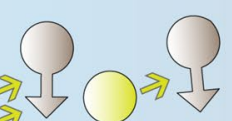

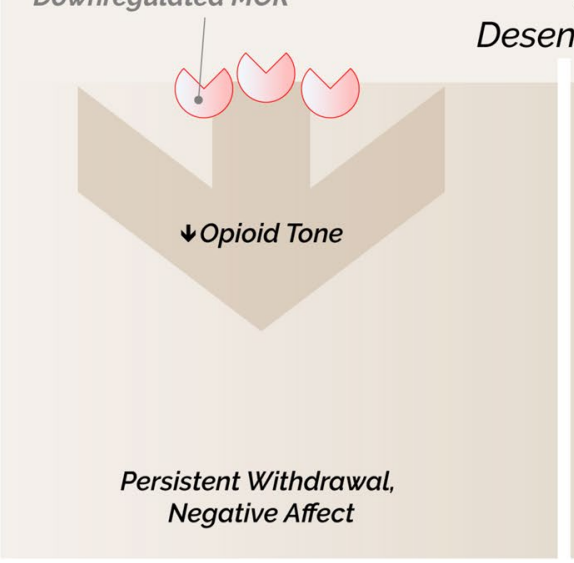

Chronic MOR Neuroadaptations

sitization and downregulation of MORs
Fig. 1 Regular interaction between buprenorphine (BUP) and full mu-opioid receptor (MOR) agonists in opioid-dependent persons. Left: chronic opioid use leads to a host of neuroadaptations at the MORs, including MOR desensitization and downregulation. These neuroadaptations are manifested clinically as a reduced opioid tone, leading to persistent opioid withdrawal, and a pervasive negative affective state. Center: full MOR agonists may temporarily increase the opioid tone, reducing opioid withdrawal and negative affect. However, the chronic MOR neuroadaptations persist, such that MORs

long-acting full opioid agonist, moderate withdrawal may not start until up to $48 \mathrm{~h}$ or longer after the last opioid dose [37]. The longer the time to start BUP, the higher the vulnerability for relapse and overdose [38].

Second, emerging evidence also suggests that high potency synthetic opioids (HSPOs), which have contaminated the illicit drug supply, especially in North America, may particularly impact induction onto BUP. HSPOs are a class of full opioid agonists-including fentanyl and its analogues - that are characterized by very high potency and lipophilicity, with intense and prolonged opioid effects [39-41]. In the USA, both clinicians and persons with OUD have reported that, with the widespread use of HSPO, remain desensitized and downregulated. Right: BUP is a partial agonist at the MOR, with low intrinsic efficacy but very high affinity. Since the affinity of BUP for the MOR is greater than that of full MOR agonists, and given its slow dissociation half-life, BUP may continue to displace full opioid agonists from MOR for up to 24-48 $\mathrm{h}$ after its dosing. Importantly, as BUP displaces full opioid agonists from MOR that are already downregulated and desensitized by chronic opioid use, this results in a profound reduction of MOR activity, thereby increasing the likelihood of precipitated withdrawal

precipitated withdrawals during inductions onto BUP have become more frequent [42].

Third, against the backdrop of the SARS-CoV-2 pandemic, major disruptions in the treatment of OUD have occurred, encouraging clinicians to attempt more inductions onto BUP at OTPs. Treatment facilities where dispensation of methadone for OUD is limited by frequent in-person monitoring, such as OTPs in the USA, have been disproportionally affected by the pandemic. Social distancing measures have inadvertently jeopardized the continuation of methadone treatment, thereby encouraging physicians to transition patients to BUP. Still, the current treatment guidelines recommend methadone to be tapered to $30-40 \mathrm{mg} /$ day, ahead 
of discontinuation. Notably, approximately $70 \%$ of persons who receive methadone for OUD do so at doses of $60 \mathrm{mg} /$ day or higher [43]. Therefore, patients are often obliged to remain on subtherapeutic doses for weeks, increasing the risk of negative outcomes [38].

Collectively, these three layers of complexity highlight the need for novel methods to induct patients onto BUP-in a safer, faster, and patient-centered manner.

\section{The Microinduction Approach}

\subsection{Definition}

In essence, microinduction involves administering small doses of BUP-typically less than $2 \mathrm{mg}$, the starting dose in standard induction strategies-such as $0.25-2 \mathrm{mg} /$ day of sublingual or $5-20 \mu \mathrm{g} / \mathrm{h}$ of transdermal BUP, with gradual increases of both the dose and frequency of administration. In contrast to the traditional induction, patients may continue their use of full opioid agonists (e.g., methadone or oxycodone), until a therapeutic dose of BUP has been achieved. At that point, the full opioid agonist is discontinued, without the need for a slow taper [44-52]. Overall, this process may take place over a 3- to 10-day period, depending on the clinical setting and level of monitoring needed; BUP microinductions in the inpatient setting may be carried out faster than in the outpatient environment [44-52].

\subsection{Pharmacological Mechanisms}

An important question is how microinduction-a slow titration of small doses of BUP that overlaps with a full opioid agonist-may allow circumventing the need for opioid-dependent persons to experience opioid withdrawal. It is believed that opioid withdrawal occurs due to a sudden decrease in opioid tone in key brain regions, including the mesolimbic area and locus coeruleus [53]. Both the reduction of MOR occupancy by full opioid agonists, as well as chronic neuroadaptations in MOR signaling as a result of prolonged opioid exposure, contribute to opioid withdrawal [54]. It is likely that microinduction reduces the risk of opioid withdrawal by affecting these mechanisms (Fig. 2).

It is conceivable that small doses of BUP administered during microinduction may stay below the threshold to displace full opioid agonists enough to induce withdrawal. For instance, sublingual maintenance doses of BUP for OUD of 2,16 , and $32 \mathrm{mg} /$ day reduced whole-brain MOR binding availability by $41 \%, 80 \%$, and $84 \%$, respectively [55]. Extrapolating from this study, the initial doses of BUP used during microinduction — as low as $0.25-1 \mathrm{mg} /$ day-are expected to displace only a small percentage of MOR. Still, with a gradual increase in BUP dose, higher quantities of full opioid agonists are expected to be displaced, resulting in withdrawal. Therefore, other mechanisms may account for the potential ability of BUP microinduction to circumvent precipitated withdrawal.

As with other GCPRs, MOR undergo several neuroadaptations in response to agonist treatment. The most notable is receptor desensitization, which results in downregulation of surface MOR, and an increase in second messenger levels [56]. Altogether, these neuroadaptations contribute to the development of opioid tolerance, dependence, and withdrawal.

Desensitization can be operationally defined as the rapid loss of MOR-effector coupling that occurs after exposure to exogenous opioids. The molecular processes underlying desensitization include rapid uncoupling of the receptor from its $\mathrm{G}$ proteins, by phosphorylation of the receptor; internalization of receptors from the cell surface; and recruitment of the beta-arrestin pathway [57]. After prolonged exposure to full opioid agonists, an eventual loss of receptor protein may occur, through changes in either the receptor degradation or synthesis.

Converging evidence indicates that the desensitization of MOR may be ligand-specific. Whereas full opioid agonists consistently desensitize the MOR [58-60], BUP does not induce desensitization-likely due to its G-protein pathway bias, with less recruitment of beta-arrestin pathway [61, 62]. Further, in contrast to MOR agonists, BUP up-regulates the number of surface MOR - an effect similar to that induced by the opioid antagonist naloxone [62]. Following long-term treatment with full opioid agonists, MOR-coupled G proteins shift from the inhibitory Gi to stimulatory Gs type, contributing to development of hyperalgesia, tolerance, and dependence [63]. Interestingly, this shift can be prevented by co-administration of with BUP along with MOR agonists [62]. Furthermore, pre-treatment with BUP has been found to block the desensitization and internalization induced by full opioid agonists [61, 64]. Collectively, these data suggest that BUP may reverse some of the neuroadaptations induced by full opioid agonists - thereby leading to resensitization. Taken together, these hypothesized mechanisms may allow maintaining an adequate opioid tone with small doses of BUP, hence preventing precipitated withdrawal—even with the co-administration of therapeutic doses of full opioid agonists (Fig. 2).

\section{Review of Microdoinduction Reports}

\subsection{Methods}

The search aimed to identify reports using the following electronic search databases: Scopus, Cochrane Database of Systematic Reviews, and Medline. The search terms 


\section{Buprenorphine Microinduction in Opioid-dependent Persons}

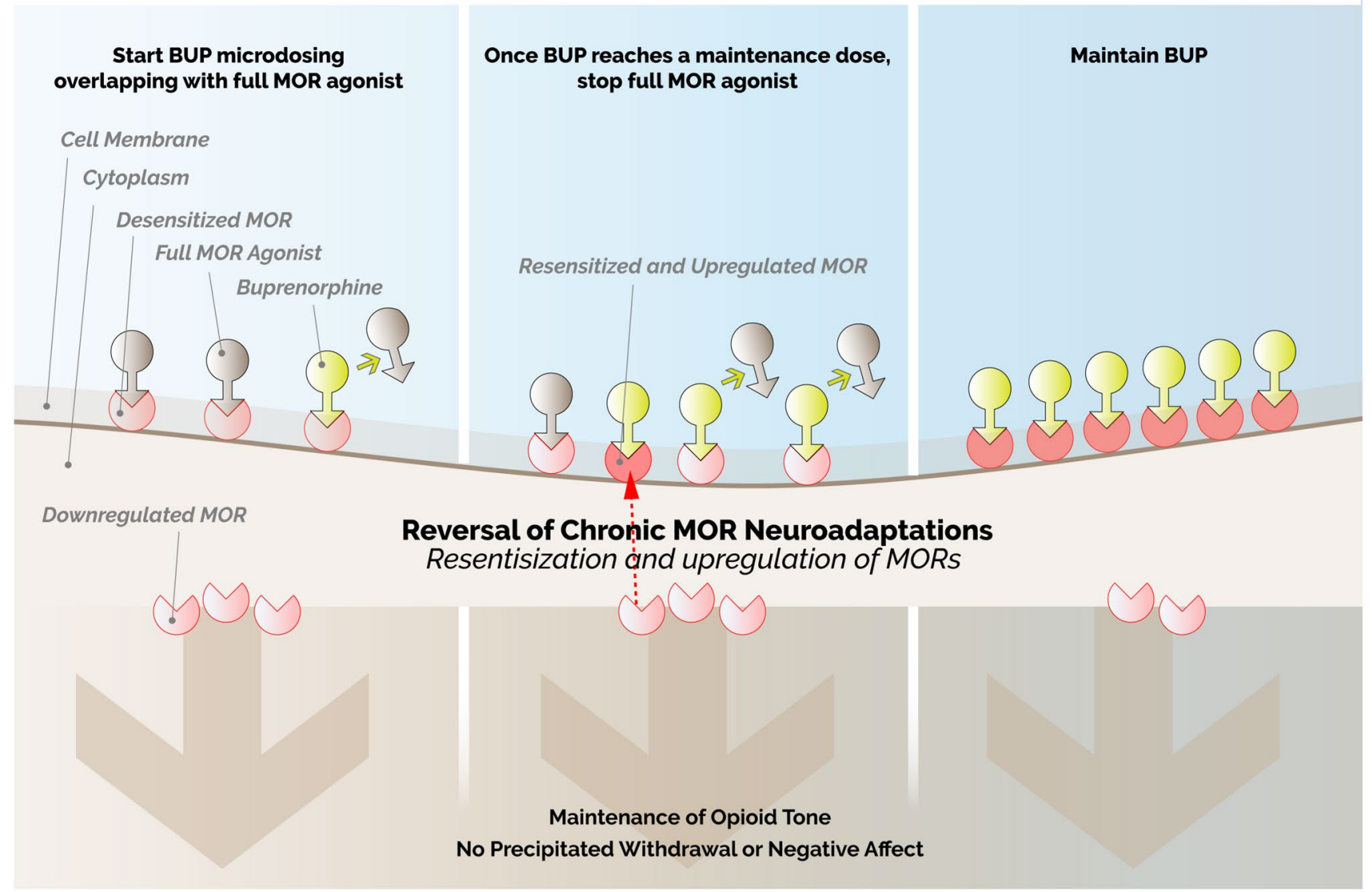

Fig. 2 Buprenorphine (BUP) microinduction in opioid-dependent persons. Left: low doses of BUP allow binding to mu-opioid receptors (MORs) below the threshold to precipitate withdrawal, maintaining the opioid tone and bypassing the worsening of negative affective states during the early phases of the treatment. Center: a gradual increase in the dose of BUP may reverse chronic MOR neuroadap-

included ([buprenorphine/naloxone, buprenorphine, suboxone] and [microdosing; induction; micro-induction, microinduction; rapid induction; Bernese method; overlap]). No date limits were included. Searches were independently conducted by J.P.D and S.P., between June and October of 2020. Reference lists from identified studies and review articles were examined to find additional studies that were not identified by the main search. Finally, we searched Clinicaltrials.gov, to ascertain whether there are ongoing randomized controlled trials investigating the efficacy of BUP microinduction.

Eligible reports included: (1) human participants undergoing BUP microinduction, and (2) the reported outcomes included opioid withdrawal. Reports were excluded if they were: (1) review papers, rather than primary literature, or (2) not peer-reviewed. tations induced by full opioid agonists. Such reversal includes an increase in the number of surface MORs, as well as MOR resensitization. Right: as full MOR agonists are discontinued, the occupation of upregulated and resensitized MOR by BUP guarantees an adequate opioid tone, circumventing the need of opioid-dependent persons to experience opioid withdrawal during the induction.

\subsection{Results}

A total of 20 reports provided data relevant to our research question (Table 1) [44-52, 65-78]. One was an open-label study, ten were case series, and nine were individual case reports. Although no published randomized controlled trials were identified, one ongoing randomized, controlled open-label superiority trial is listed in Clinicaltrials.gov; this unpublished study was designed to compare safety and effectiveness of buprenorphine microinduction versus standard induction among persons with OUD, and its results remain unavailable.

The available reports differed with regards to: (1) BUP formulations (e.g., transdermal system, sublingual tablets/ films) were used; (2) the starting point of the microinduction - the full opioid agonist dose, expressed in morphine milligram equivalency (MME); (3) the speed of BUP 
titration; (4) the time point when the full opioid agonist was discontinued; (5) presence of either pain and/or OUD in the sample; (6) use of ancillary medications for opioid withdrawal; (7) other clinical outcomes, such as non-medical opioid use.

\section{Discussion}

The most notable finding of this review was that most of the data available are derived from either open-label studies or case series-reflecting the novelty of the BUP microinduction approach, as well as enduring adherence to traditional induction protocols. Despite the lack of systematic, highquality evidence to support its use, all reports indicate that BUP microinduction was safe and well tolerated, providing early signs for its feasibility. Taken together, the pharmacological plausibility of BUP microinduction, coupled with its promise to increase access to this first-line treatment for OUD, highlight the timely need for randomized, placebocontrolled studies to establish the safety and efficacy of this approach. The contributions of this review include mechanistic, methodological, and clinical implications.

\subsection{Mechanistic Implications}

At the mechanistic level, we have underscored the need to uncover how BUP affects MOR and their signaling, especially against the backdrop of full opioid agonist-induced neuroadaptations. At present, the level of MOR receptor occupancy required to produce precipitated withdrawal by BUP remains to be investigated in preclinical and clinical studies. For instance, there are no positron emission tomography (PET) studies examining the impact of microdoses of BUP among individuals receiving long-term, high-dose full opioid agonist therapy. Clarifying the level of MOR receptor occupancy that is sufficient to provide therapeutic benefit while avoiding precipitated withdrawal may guide the dose range that is optimal for BUP microinduction. Moreover, the capacity of BUP to reverse clinically relevant neuroadaptations induced by full opioid agonists-desensitization, downregulation, and shift to stimulatory Gs type of G proteins-resemble earlier studies that used very low doses of naltrexone combined with full opioid agonists, and likewise warrants further research [79]. Future preclinical and translational studies are required to elucidate the specific mechanisms involved in microinduction.

\subsection{Methodological Implications}

Despite its growing popularity, BUP microinduction has not yet been systematically examined in well-controlled studies, which explains the wide variability of methods and outcomes included in the existing reports.

For patients with OUD, the main clinically relevant outcomes of BUP microinduction include: (1) Successful completion of the microinduction regimen; (2) retention in treatment, using a longer-term, well-defined timepoint of at least 6 months [80-82]; (3) change in non-medical opioid use; (4) emergence of adverse effects, such as precipitated opioid withdrawal, or mortality by opioid overdose. Further, future studies should also include assessments of patient comfort, healthcare provider experience, and ease of administering microinduction protocols.

Several other methodological questions in BUP microinduction warrant further consideration. First, the optimal time to discontinue the full opioid agonist remains understudied. For instance, it is possible that different full opioid agonists (e.g., methadone, oxycodone) may need to be discontinued at different time points during the microinduction, based on their pharmacodynamic (e.g., ligand-specific effects) or pharmacokinetic (e.g., differences in half-life) properties. Second, the role of patient expectancy in mediating the success of microinduction should not be understated. It is likely that positive expectations about the microinduction process impact the likelihood of completing it, and vice versa. Thus, patients who did not tolerate BUP microinduction may have been excluded from case series-which, thus far, still constitute the bulk of the evidence supporting this approach. Adequate blinding, using randomized, placebo-controlled, double-dummy designs may account for the conceivably high impact of expectancy on the short-term outcomes of BUP microinduction. The lack of methodologically sound trials remains the largest barrier to widespread implementation of this approach.

\subsection{Clinical Implications}

The main clinical implication of BUP microinduction is its potential to enhance patient engagement with pharmacotherapy for OUD in a timely and efficient manner.

The reports included in this review had individuals receiving a wide range of opioid doses, including doses of over $1000 \mathrm{mg}$ /day of MME. Even at high full opioid agonist doses, the opioid withdrawal severity during microinduction remained mild, with most participants successfully completing the induction. Persons with OUD receiving long-term and high doses of full opioid agonists-for example, $>90$ MME/day for 3 months or longer-present a particular challenge for induction onto BUP. Patients with chronic pain on long-term opioid therapy are also physiologically dependent on opioids, and their challenges during induction onto BUP overlap with those of patients with OUD. Hence, these early findings provide preliminary support for microinduction as a potential strategy for patients receiving a wide dose 


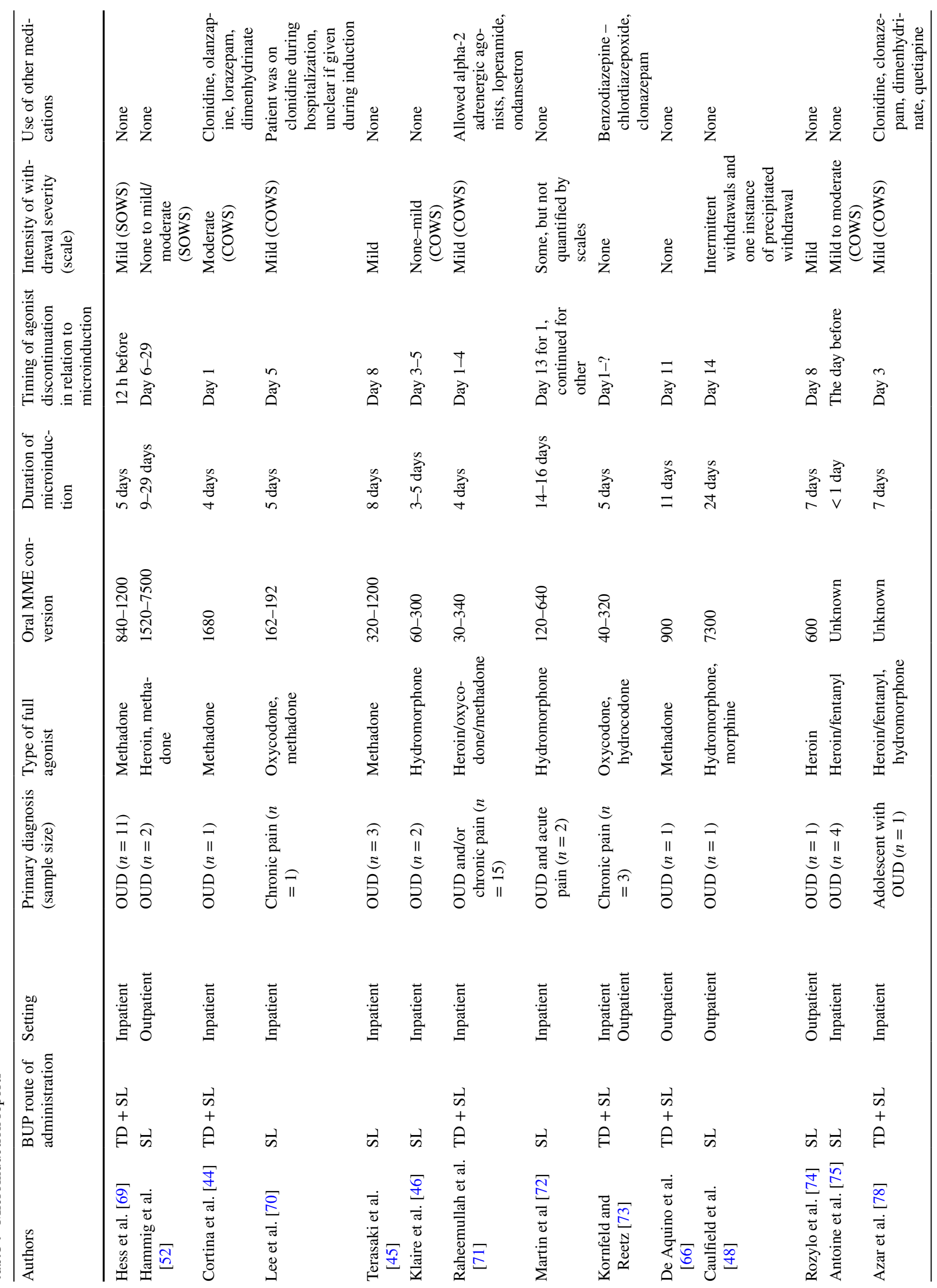




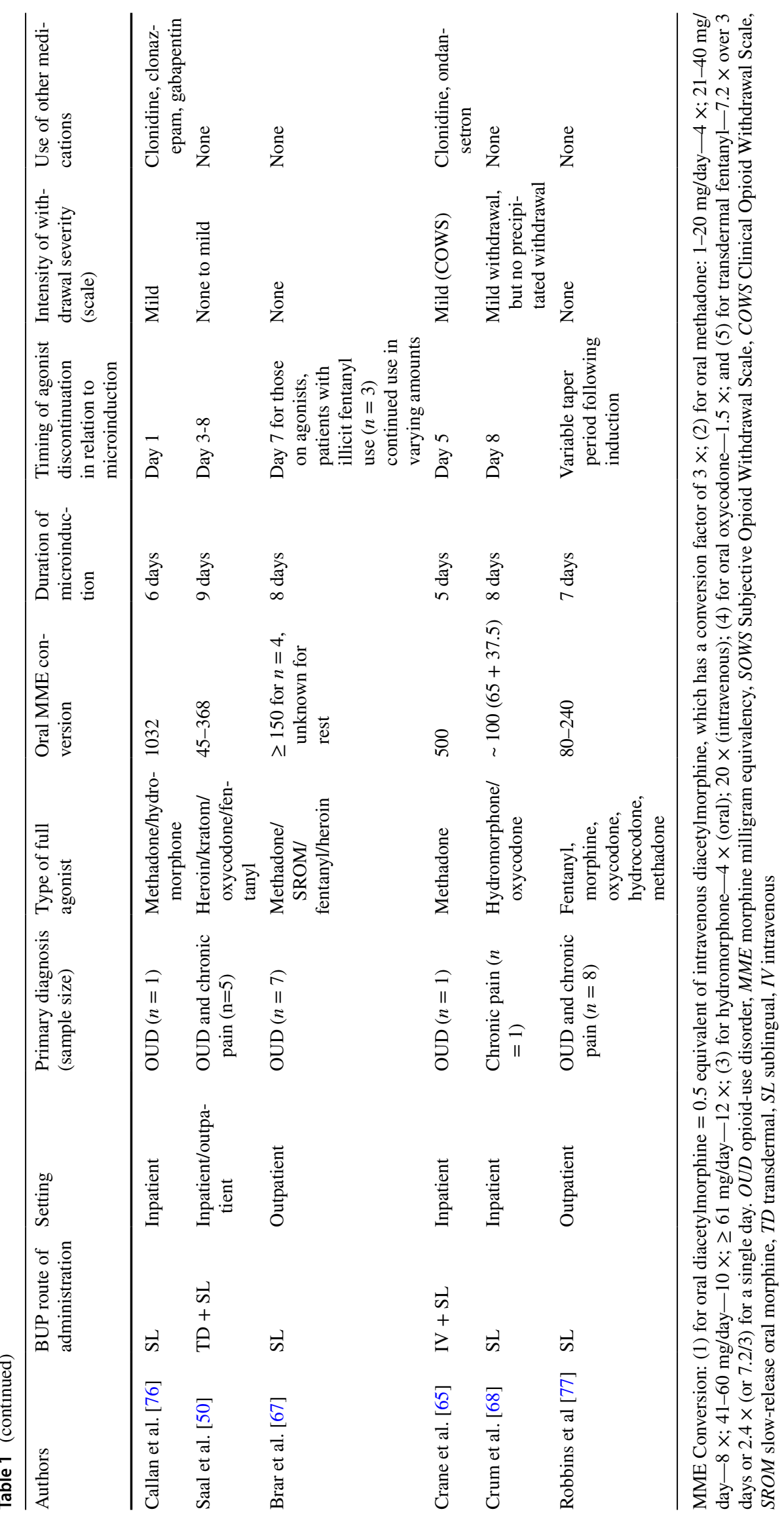


range of long-term opioid treatment for distinct indications. Variations in microinduction protocols may be appropriate depending on the patient population (OUD vs. chronic pain receiving long-term opioid therapy) and clinical setting (inpatient vs. outpatient).

Future well-controlled studies should examine the optimum dose, route of administration - sublingual versus transcutaneous - as well as the duration of BUP microinduction. It is possible that distinct microinduction approaches represent different ways of exploiting differences in the pharmacological profile of opioids to improve treatment outcomes.

\section{Conclusions}

Despite overwhelming evidence supporting the effectiveness of BUP pharmacotherapy for curbing fatalities among persons with OUD, the ceiling effect of BUP for opioid overdose, and the office-based prescribing of BUP in the USA, the requirement of moderate opioid withdrawal is still a significant barrier for induction. This requirement is, in part, a clinical manifestation of chronic neuroadaptations induced by full opioid agonists-MOR desensitization and downregulation - which may be partly reversed by BUP, in a dose-dependent manner. By harnessing the idiosyncratic pharmacological profile of BUP, microinduction holds promise to increase access to BUP pharmacotherapy and improve treatment outcomes. While mechanistic and clinical reports provide preliminary evidence supporting this innovative approach, further translational research, as well as welldesigned randomized, placebo-controlled studies, are needed to ascertain whether the potential of BUP microinduction can be fully realized.

Acknowledgements The authors acknowledge the VISN-1 VA Mental Illness, Research, and Education Clinical Center (MIRECC); the Robert E. Leet and Clara Guthrie Patterson Trust; and the National Institute of Drug Abuse (R25DA033211-07). Mentored Clinical Research Award, National Institute on Drug Abuse, R25DA033211-07, U.S. Department of Veterans Affairs, MIRECC VISN-1.

\section{Declarations}

Funding JPD is supported by the VISN-1 VA Mental Illness, Research, and Education Clinical Center (MIRECC), the Robert E. Leet and Clara Guthrie Patterson Trust, and the National Institute of Drug Abuse (R25DA033211-07).

Conflicts of interest/Competing interests The authors have no conflicts of interest to report.

Ethics approval Not applicable.

Consent to participate Not applicable.

Consent for publication Not applicable.
Availability of data and material Not applicable.

Code availability Not applicable.

Authors' contributions J.P.D. and M.S. were responsible for the concept and design of the review. J.P.D. and S.P. performed independent review of the clinical reports. MS assisted with interpretation of findings. J.P.D. and M.S. drafted the manuscript. S.P. provided critical revision of the manuscript with important intellectual content. All authors critically reviewed content and approved the final version for publication.

\section{References}

1. Degenhardt L, Whiteford HA, Ferrari AJ, Baxter AJ, Charlson FJ, Hall WD, et al. Global burden of disease attributable to illicit drug use and dependence: findings from the Global Burden of Disease Study 2010. Lancet. 2013;382(9904):1564-74.

2. Hser Y-I, Evans E, Grella C, Ling W, Anglin D. Long-term course of opioid addiction. Harv Rev Psychiatry. 2015;23(2):76-89.

3. Blanco C, Volkow ND. Management of opioid use disorder in the USA: present status and future directions. Lancet. 2019;393(10182):1760-72.

4. Larochelle MR, Bernson D, Land T, Stopka TJ, Wang N, Xuan $\mathrm{Z}$, et al. Medication for opioid use disorder after nonfatal opioid overdose and association with mortality: a cohort study. Ann Internal Med. 2018;169(3):1637-2145.

5. Cowan A, Lewis J, Macfarlane I. Agonist and antagonist properties of buprenorphine, a new antinociceptive agent. Br J Pharmacol. 1977;60(4):537-45.

6. Phillips JK, Ford MA, Bonnie RJ. Trends in opioid use, harms, and treatment. In: Pain Management and the opioid epidemic: balancing societal and individual benefits and risks of prescription opioid use. Washington: National Academies Press; 2017.

7. Williams AR, Nunes EV, Bisaga A, Pincus HA, Johnson KA, Campbell AN, et al. Developing an opioid use disorder treatment cascade: a review of quality measures. J Subst Abuse Treat. 2018;91:57-68.

8. Whitley SD, Sohler NL, Kunins HV, Giovanniello A, Li X, Sacajiu G, et al. Factors associated with complicated buprenorphine inductions. J Subst Abuse Treat. 2010;39(1):51-7.

9. Dunn KE, Weerts EM, Huhn AS, Schroeder JR, Tompkins DA, Bigelow GE, et al. Preliminary evidence of different and clinically meaningful opioid withdrawal phenotypes. Addict Biol. 2020;25(1):e12680.

10. Becker WC, Fiellin DA. When epidemics collide: coronavirus disease 2019 (COVID-19) and the opioid crisis. American College of Physicians; 2020.

11. Sordo L, Barrio G, Bravo MJ, Indave BI, Degenhardt L, Wiessing $\mathrm{L}$, et al. Mortality risk during and after opioid substitution treatment: systematic review and meta-analysis of cohort studies. BMJ. 2017;357:j1550.

12. Ghosh SM, Klaire S, Tanguay R, Manek M, Azar P. A review of novel methods to support the transition from methadone and other full agonist opioids to buprenorphine/naloxone sublingual in both community and acute care settings. Can J Addict. 2019;10(4):41-50.

13. Moe J, Doyle-Waters MM, O'Sullivan F, Hohl CM, Azar P. Effectiveness of micro-induction approaches to buprenorphine initiation: a systematic review protocol. Addict Behav. 2020;111:106551. 
14. Khanna IK, Pillarisetti S. Buprenorphine—an attractive opioid with underutilized potential in treatment of chronic pain. J Pain Res. 2015;8:859.

15. Kress HG. Clinical update on the pharmacology, efficacy and safety of transdermal buprenorphine. Eur J Pain. 2009;13(3):219-30.

16. Lewis J. Ring C-bridged derivatives of thebaine and oripavine. Adv Biochem Psychopharmacol. 1973;8:123.

17. Matthes HW, Maldonado R, Simonin F, Valverde O, Slowe S, Kitchen I, et al. Loss of morphine-induced analgesia, reward effect and withdrawal symptoms in mice lacking the $\mu$-opioid-receptor gene. Nature. 1996;383(6603):819.

18. Filliol D, Ghozland S, Chluba J, Martin M, Matthes HW, Simonin $F$, et al. Mice deficient for $\delta$-and $\mu$-opioid receptors exhibit opposing alterations of emotional responses. Nat Genet. 2000;25(2):195.

19. Spanagel R, Herz A, Shippenberg TS. The effects of opioid peptides on dopamine release in the nucleus accumbens: an in vivo microdialysis study. J Neurochem. 1990;55(5):1734-40.

20. Sutcliffe KJ, Henderson G, Kelly E, Sessions RB. Drug binding poses relate structure with efficacy in the $\mu$ opioid receptor. J Mol Biol. 2017;429(12):1840-51.

21. Bodkin JA, Zornberg GL, Lukas SE, Cole JO. Buprenorphine treatment of refractory depression. J Clin Psychopharmacol. 1995;15(1):49-57.

22. Huang P. Comparison of pharmacological activities of buprenorphine and norbuprenorphine: norbuprenorphine is a potent opioid agonist. J Pharmacol Exp Ther. 2001;297(2):688-95.

23. Volpe DA. Uniform assessment and ranking of opioid Mu receptor binding constants for selected opioid drugs. Regul Toxicol Pharmacol. 2011;59(3):385-90.

24. Zuurmond W, Meert T, Noorduin H. Partial versus full agonists for opioid-mediated analgesia-focus on fentanyl and buprenorphine. Acta Anaesthesiol Belg. 2002;53(3):193.

25. Walsh SL. Clinical pharmacology of buprenorphine: ceiling effects at high doses. Clin Pharmacol Ther. 1994;55(5):569-80.

26. Martin WR, Eades C, Thompson J, Huppler R, Gilbert P. The effects of morphine-and nalorphine-like drugs in the nondependent and morphine-dependent chronic spinal dog. J Pharmacol Exp Ther. 1976;197(3):517-32.

27. Corder G, Castro DC, Bruchas MR, Scherrer G. Endogenous and exogenous opioids in pain. Annu Rev Neurosci. 2018;41:453-73.

28. Pathan $\mathrm{H}$, Williams J. Basic opioid pharmacology: an update. $\mathrm{Br}$ J Pain. 2012;6(1):11-6.

29. Schmid CL, Kennedy NM, Ross NC, Lovell KM, Yue Z, Morgenweck J, et al. Bias factor and therapeutic window correlate to predict safer opioid analgesics. Cell. 2017;171(5):1165-75.

30. Cahill CM, Walwyn W, Taylor AM, Pradhan AA, Evans CJ. Allostatic mechanisms of opioid tolerance beyond desensitization and downregulation. Trends Pharmacol Sci. 2016;37(11):963-76.

31. Whistler JL, Chuang H-H, Chu P, Jan LY, Von Zastrow M. Functional dissociation of $\mu$ opioid receptor signaling and endocytosis: implications for the biology of opiate tolerance and addiction. Neuron. 1999;23(4):737-46.

32. Lau EK, Trester-Zedlitz M, Trinidad JC, Kotowski SJ, Krutchinsky AN, Burlingame AL, et al. Quantitative encoding of the effect of a partial agonist on individual opioid receptors by multisite phosphorylation and threshold detection. Sci Signal. 2011;4(185):ra52.

33. Bell JR, Butler B, Lawrance A, Batey R, Salmelainen P. Comparing overdose mortality associated with methadone and buprenorphine treatment. Drug Alcohol Depend. 2009;104(1-2):73-7.

34. Fareed A. Hypogonadism and low testosterone levels as a side effect of methadone and buprenorphine. Int J High Risk Behav Addict. 2012;1(2):84.
35. Kelty E, Hulse G. Fatal and non-fatal opioid overdose in opioid dependent patients treated with methadone, buprenorphine or implant naltrexone. Int J Drug Policy. 2017;46:54-60.

36. Rosado J, Walsh SL, Bigelow GE, Strain EC. Sublingual buprenorphine/naloxone precipitated withdrawal in subjects maintained on $100 \mathrm{mg}$ of daily methadone. Drug Alcohol Depend. 2007;90(2-3):261-9.

37. Center for Substance Abuse Treatment. Clinical Guidelines for the Use of Buprenorphine in the Treatment of Opioid Addiction. Rockville (MD): Substance Abuse and Mental Health Services Administration (US); 2004. (Treatment Improvement Protocol (TIP) Series, No. 40.) Available from: https://www.ncbi.nlm.nih. gov/books/NBK64245/. Accessed 29 Mar 2021.

38. Termorshuizen F, Krol A, Prins M, Geskus R, van den Brink W, van Ameijden EJ. Prediction of relapse to frequent heroin use and the role of methadone prescription: an analysis of the Amsterdam Cohort Study among drug users. Drug Alcohol Depend. 2005;79(2):231-40.

39. Armenian P, Vo KT, Barr-Walker J, Lynch KL. Fentanyl, fentanyl analogs and novel synthetic opioids: a comprehensive review. Neuropharmacology. 2018;134:121-32.

40. McClain DA, Hug CC Jr. Intravenous fentanyl kinetics. Clin Pharmacol Ther. 1980;28(1):106-14.

41. Maguire P, Tsai N, Kamal J, Cometta-Morini C, Upton C, Loew G. Pharmacological profiles of fentanyl analogs at $\mu, \delta$ and $\kappa$ opiate receptors. Eur J Pharmacol. 1992;213(2):219-25.

42. Silverstein SM, Daniulaityte R, Martins SS, Miller SC, Carlson RG. "Everything is not right anymore": buprenorphine experiences in an era of illicit fentanyl. Int J Drug Policy. 2019;74:76-83.

43. D'Aunno T, Pollack HA. Changes in methadone treatment practices: results from a national panel study, 1988-2000. JAMA. 2002;288(7):850-6.

44. Cortina S, Mihic T, Fennemore M, McLean M. Case report: highdose methadone transition to buprenorphine/naloxone in an Inpatient with a prolonged QT interval. Can J Addict. 2017;8(1):25-8.

45. Terasaki D, Smith C, Calcaterra SL. Transitioning hospitalized patients with opioid use disorder from methadone to buprenorphine without a period of opioid abstinence using a microdosing protocol. Pharmacotherapy. 2019;39:1023-9.

46. Klaire S, Zivanovic R, Barbic SP, Sandhu R, Mathew N, Azar P. Rapid micro-induction of buprenorphine/naloxone for opioid use disorder in an inpatient setting: a case series. Am J Addict. 2019;28:262-5.

47. Ward HB, Barnett BS, Suzuki J. Rapid transition from methadone to buprenorphine using naltrexone-induced withdrawal: a case report. Subst Abuse. 2019;40:1-6.

48. Caulfield MDG, Brar R, Sutherland C, Nolan S. Transitioning a patient from injectable opioid agonist therapy to sublingual buprenorphine/naloxone for the treatment of opioid use disorder using a microdosing approach. BMJ Case Rep. 2020;13(3):e233715.

49. Randhawa PA, Brar R, Nolan S. Buprenorphine-naloxone "microdosing": an alternative induction approach for the treatment of opioid use disorder in the wake of North America's increasingly potent illicit drug market. Can Med Assoc J. 2020;192(3):E73.

50. Saal D, Lee F. Rapid induction therapy for opioid-use disorder using buprenorphine transdermal patch: a case series. Perm J. 2020;24:19.

51. Tang VM, Lam-Shang-Leen J, Brothers TD, Hansen K, Caudarella A, Lamba W, et al. Case series: limited opioid withdrawal with use of transdermal buprenorphine to bridge to sublingual buprenorphine in hospitalized patients. Am J Addict. 2020;29(1):73.

52. Hämmig R, Kemter A, Strasser J, von Bardeleben U, Gugger B, Walter $\mathrm{M}$, et al. Use of microdoses for induction of buprenorphine 
treatment with overlapping full opioid agonist use: the Bernese method. Su-hak. 2016;7:99.

53. Cahill CM, Walwyn W, Taylor AMW, Pradhan AAA, Evans CJ. Allostatic mechanisms of opioid tolerance beyond desensitization and downregulation. Trends Pharmacol Sci. 2016;37(11):963-76.

54. Rehni AK, Jaggi AS, Singh N. Opioid withdrawal syndrome: emerging concepts and novel therapeutic targets. CNS Neurol Disord Drug Targets. 2013;12(1):112-25.

55. Greenwald MK, Johanson CE, Moody DE, Woods JH, Kilbourn MR, Koeppe RA, et al. Effects of buprenorphine maintenance dose on mu-opioid receptor availability, plasma concentrations, and antagonist blockade in heroin-dependent volunteers. Neuropsychopharmacology. 2003;28(11):2000-9.

56. Böhm SK, Grady EF, Bunnett NW. Regulatory mechanisms that modulate signalling by G-protein-coupled receptors. Biochem J. 1997;322(1):1-18.

57. Dang VC, Christie MJ. Mechanisms of rapid opioid receptor desensitization, resensitization and tolerance in brain neurons. Br J Pharmacol. 2012;165(6):1704-16.

58. Arden JR, Segredo V, Wang Z, Lameh J, Sadée W. Phosphorylation and agonist-specific intracellular trafficking of an epitopetagged $\mu$-opioid receptor expressed in HEK 293 cells. J Neurochem. 1995;65(4):1636-45.

59. Sternini C, Spann M, Anton B, Keith DE, Bunnett NW, Von Zastrow M, et al. Agonist-selective endocytosis of mu opioid receptor by neurons in vivo. Proc Natl Acad Sci. 1996;93(17):9241-6.

60. Keith DE, Anton B, Murray SR, Zaki PA, Chu PC, Lissin DV, et al. $\mu$-Opioid receptor internalization: opiate drugs have differential effects on a conserved endocytic mechanism in vitro and in the mammalian brain. Mol Pharmacol. 1998;53(3):377-84.

61. Virk MS, Arttamangkul S, Birdsong WT, Williams JT. Buprenorphine is a weak partial agonist that inhibits opioid receptor desensitization. J Neurosci. 2009;29(22):7341-8.

62. Zaki PA, Keith DE, Brine GA, Carroll FI, Evans CJ. Ligandinduced changes in surface $\mu$-opioid receptor number: relationship to $\mathrm{G}$ protein activation? J Pharmacol Exp Ther. 2000;292(3):1127-34.

63. Largent-Milnes TM, Guo W, Wang HY, Burns LH, Vanderah TW. Oxycodone plus ultra-low-dose naltrexone attenuates neuropathic pain and associated mu-opioid receptor-Gs coupling. J Pain. 2008;9(8):700-13.

64. Yu Y, Zhang L, Yin X, Sun H, Uhl GR, Wang JB. Mu opioid receptor phosphorylation, desensitization, and ligand efficacy. J Biol Chem. 1997;272(46):28869-74.

65. Crane K, Snead J, Stanley R, Avery J, Ghosh SM, Mints G. Intravenous Buprenorphine Micro-dosing Induction in a Patient on Methadone Treatment: A Case Report. Psychosomatics. 2020;S0033-3182(20):30213-9. https://doi.org/10.1016/j.psym. 2020.07.004

66. De Aquino JP, Fairgrieve C, Klaire S, Garcia-Vassallo G. Rapid Transition From Methadone to Buprenorphine Utilizing a Microdosing Protocol in the Outpatient Veteran Affairs Setting. J Addict Med. 2020;14(5):e271-3. https://doi.org/10.1097/ADM.00000 00000000618 .

67. Brar R, Fairbairn N, Sutherland C, Nolan S. Use of a novel prescribing approach for the treatment of opioid use disorder: Buprenorphine/naloxone micro-dosing-a case series. Drug Alcohol Rev. 2020;39(5):588-94.
68. Crum IT, Karre VMM, Balasanova AA. Transitioning from intrathecal hydromorphone to sublingual buprenorphinenaloxone through microdosing: a case report. A\&A Pract. 2020;14(11):e01316.

69. Hess M, Boesch L, Leisinger R, Stohler R. Transdermal buprenorphine to switch patients from higher dose methadone to buprenorphine without severe withdrawal symptoms. Am J Addict. 2011;20(5):480-1.

70. Lee DS, Hann JE, Klaire SS, Nikoo M, Negraeff MD, RezazadehAzar P. Rapid induction of buprenorphine/naloxone for chronic pain using a microdosing regimen: a case report. A\&A Pract. 2020;14(2):44-7.

71. Raheemullah A, Lembke A. Buprenorphine Induction Without Opioid Withdrawal: A Case Series of 15 Opioid-Dependent Inpatients Induced on Buprenorphine Using Microdoses of Transdermal Buprenorphine. Am J Ther. 2019. https://doi.org/10.1097/ MJT.0000000000001108.

72. Martin L, Lennox R, Regenstreif L, O'Shea T. Case Report: "Striving to Skip the Withdrawal" using buprenorphinenaloxone microdosing for hospitalized patients. Can J Addict. 2019;10(4):35-40.

73. Kornfeld H, Reetz H. Transdermal buprenorphine, opioid rotation to sublingual buprenorphine, and the avoidance of precipitated withdrawal: a review of the literature and demonstration in three chronic pain patients treated with butrans. Am J Ther. 2015;22(3):199-205.

74. Rozylo J, Mitchell K, Nikoo M, Durante SE, Barbic SP, Lin D, et al. Case report: successful induction of buprenorphine/naloxone using a microdosing schedule and assertive outreach. Addict Sci Clin Pract. 2020;15(1):1-6.

75. Antoine D, Huhn AS, Strain EC, Turner G, Jardot J, Hammond AS, Dunn KE. Method for Successfully Inducting Individuals Who Use Illicit Fentanyl Onto Buprenorphine/Naloxone. Am J Addict. 2021;30(1):83-7. https://doi.org/10.1111/ajad.13069.

76. Callan J, Pytell J, Ross J, Rastegar DA. Transition from methadone to buprenorphine using a short-acting agonist bridge in the inpatient setting: a case study. J Addict Med. 2020;14(5):e274-6.

77. Robbins JL, Englander H, Gregg J. Buprenorphine Microdose Induction for the Management of Prescription Opioid Dependence. J Am Board Fam Med. 2021;34(Suppl):S141-6. https://doi. org/10.3122/jabfm.2021.S1.200236.

78. Azar P, Wong JSH, Jassemi S, Moore E, Vo DX, Nikoo M, Young S. A Case Report: Rapid Micro-Induction of Buprenorphine/ Naloxone to Administer Buprenorphine Extended-Release in an Adolescent With Severe Opioid Use Disorder. Am J Addict. 2020;29(6):531-5. https://doi.org/10.1111/ajad.13050.

79. Kim PS, Fishman MA. Low-dose naltrexone for chronic pain: update and systemic review. Curr Pain Headache Rep. 2020;24(10):64.

80. Timko C, Schultz NR, Cucciare MA, Vittorio L, Garrison-Diehn C. Retention in medication-assisted treatment for opiate dependence: a systematic review. J Addict Dis. 2016;35(1):22-35.

81. Gossop M, Marsden J, Stewart D, Kidd T. The National Treatment Outcome Research Study (NTORS): 4-5 year follow-up results. Addiction. 2003;98(3):291-303.

82. Hser Y-I, Hoffman V, Grella CE, Anglin MD. A 33-year follow-up of narcotics addicts. Arch Gen Psychiatry. 2001;58(5):503-8. 